\title{
Étude préliminaire sur la prévalence de Campylobacter dans les diarrhées infantiles au nord du Liban
}

\author{
F. Dabboussi, ${ }^{1,2}$ S. Alam, ' H. Mallat, ${ }^{1,2}$ S. Hlais ${ }^{1,2}$ et M. Hamze ${ }^{1,2}$
}

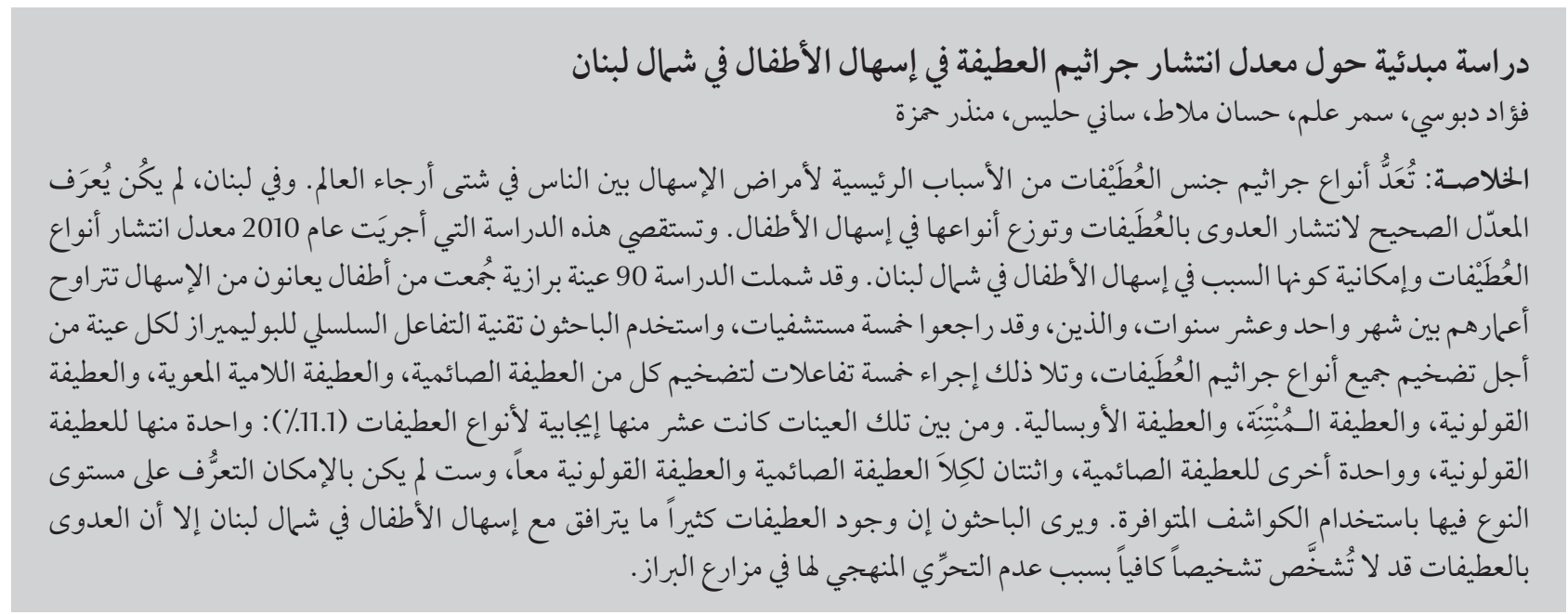

RÉSUMÉ Les espèces du genre Campylobacter sont une cause majeure de maladies diarrhéiques humaines dans le monde. Au Liban, la prévalence réelle des infections à Campylobacter ainsi que la distribution des espèces dans les diarrhées infantiles ne sont pas connues. Cette étude menée en 2010 a examiné la prévalence de Campylobacter et le rôle étiologique qu'il peut jouer dans la diarrhée infantile au nord du Liban. Au total, 90 échantillons de selles d'enfants (âgés de 1 mois à 10 ans) présentant une diarrhée ont été recueillis auprès de cinq hôpitaux. La technique de réaction de polymérisation en chaîne (PCR) a été utilisée pour chaque échantillon pourl'amplification de toutes les espèces de Campylobacter, suivie par cinq réactions de PCR pour l'amplification de C. jejuni, C. hyointestinalis, C. coli,C. fetus et $C$. upsaliensis.Surles90échantillons, 10 ontétépositifspourCampylobacterspp(11,1\%):1pour $C$. coli, 1 pour $C$.jejuni, 2 pour $C$. jejuni et $C$. coli à la fois, et 6 n'ont pas pu être identifiés au rang de l'espèce avec les amorces spécifiques. Campylobacter peut souvent être associée à la diarrhée infantile au nord du Liban mais I'infection à Campylobacter peut être considérablement sous-diagnostiquée étant donné l'absence de recherche systématique de Campylobacter dans les coprocultures.

\section{Preliminary study on the prevalence of Campylobacter in childhood diarrhoea in north Lebanon}

ABSTRACT Campylobacter species are a major cause of human diarrhoeal disease worldwide. In Lebanon, the true prevalence of Campylobacter infections and the species distribution in childhood diarrhoea are not known. This study in 2010 investigated the prevalence of Campylobacter species and its possible etiologic role in childhood diarrhoea in north Lebanon. A total of 90 stool samples from children (aged 1 month to 10 years) presenting with diarrhoea were collected from 5 hospitals. A polymerase chain reaction technique (PCR) was used for each sample for the amplification of all Campylobacter species followed by 5 PCR reactions for the amplification of $C$. jejuni, C. hyointestinalis, $C$. coli, $C$. fetus and C. upsaliensis. Of the 90 samples, 10 were positive for Campylobacter species (11.1\%): 1 for C.coli, 1 for C.jejuni, 2 for both $C$. jejuni and $C$. coli, and 6 could not be identified to the species level with the available primers. Campylobacter species is frequently associated with childhood diarrhoea in north Lebanon but Campylobacter infection may be significantly underdiagnosed because the search for Campylobacter is not part of the routine stool culture.

'Laboratoire Microbiologie santé et environnement, Centre Azm pour la Recherche en Biotechnologie et ses Applications, École doctorale des Sciences et de Technologie, Université Libanaise, Tripoli (Liban).

${ }^{2}$ Faculté de santé publique, Université Libanaise, Tripoli (Liban)(Correspondance à adresserà M. Hamze : mhamze@monzerhamze.com). Reçu : 16/02/12 accepté : 26/02/12 


\section{Introduction}

Le genre Campylobacter comprend nombre d'espèces [1]. Les microorganismes de ce genre sont typiquement des bactéries à coloration de Gram négative, asporulées, en forme de $S$ ou de spirale $(0,2$ à $0,8 \mu \mathrm{m}$ de large et 0,5 à $5 \mu \mathrm{m}$ de long) avec un seul flagelle polaire à l'une ou aux deux extrémités, leur conférant une mobilité caractéristique en vrille. Ces bactéries sont micro-aérophiles mais certaines peuvent également pousser en aérobiose ou en anaérobiose. Quelques espèces, en particulier C. jejuni, C. coli et C. lari, sont thermotolérantes avec un optimum de croissance à $42{ }^{\circ} \mathrm{C}$. Elles peuvent coloniser les muqueuses, d'ordinaire le tractus intestinal de la plupart des espèces testées de mammifères et d'oiseaux [1].

Les bactéries du genre Campylobacter sont la cause principale de maladies intestinales humaines d'origine bactérienne aussi bien dans les pays développés, où son incidence augmente, que dans les pays en développement [2].

Plus de $80 \%$ des cas de campylobactériose sont causés par C. jejuni et $10 \%$ des cas sont causés par C. coli.D'autres espèces de Campylobacter, telles que $C$. concisus, $C$. upsaliensi, $C$. lari et C. fetus, peuvent également être associées à des diarrhées chez l'homme [3].

La source primaire des infections à $C$. jejuni ou $C$. coli chez l'homme est supposée être la manipulation ou la consommation de viandes contaminées, en particulier la viande de volaille. Cependant les contacts avec les animaux de compagnie et le bétail, la consommation d'eau contaminée ou de lait cru et les voyages dans les zones à forte prévalence sont aussi considérés comme des facteurs de risque de la maladie humaine [4]. Le contrôle de Campylobacter dans la chaîne alimentaire est maintenant devenu une cible majeure des agences en charge de la sécurité alimentaire dans le monde.
Les bactéries du genre Campylobacter sont très exigeantes, et les agents antimicrobiens incorporés dans les milieux de culture utilisés pour isoler sélectivement C. jejuni et C. coli ont été capables d'inhiber la croissance d'autres espèces de Campylobacter, telles que C. upsaliensis, C. hyointestinalis, et C.fetus. En conséquence, les méthodes microbiologiques ne fournissent pas une mesure réelle de la fréquence et de la diversité des espèces de Campylobacter. L'application des méthodes moléculaires telle que la réaction de polymérisation en chaîne (PCR) peut fournir une description plus précise de la prévalence des espèces de Campylobacter [5].

L'objectif de cette étude était d'établir par PCR la prévalence de Campylobacter chez les enfants diarrhéiques au nord du Liban d'une part, et d'autre part de déterminer la distribution des différentes espèces impliquées.

\section{Méthodes}

L'étude a eu lieu au Centre AZM pour la Recherche en Biotechnologie et ses Applications entre le 7 avril et le 15 juin 2010. Au total, 90 échantillons de selles d'enfants diarrhéiques ont été collectés auprès de cinq hôpitaux au nord du Liban (Hôpital Nini, Hôpital gouvernemental de Tripoli, Hôpital de Batroun, Hôpital Haykal et Hôpital Mazloum). Les échantillons de selles avaient été conservés à $-20^{\circ} \mathrm{C}$.

\section{Extraction de l'ADN}

L'extraction de l'ADN à partir des matières fécales humaines a été réalisée en utilisant le kit QIAamp DNA Stool Mini Kit (QIAGEN) selon les instructions fournies par le fabricant. Le QIAamp DNA Stool Mini Kit permet la purification rapide de l'ADN à partir de selles humaines fraîches ou congelées. Ce kit est composé d'une solution de lyse. Les impuretés et les inhibiteurs de la réaction PCR sont éliminés par une résine d'adsorption. Ensuite l'ADN est purifié sur des colonnes QIAamp MiniSpin.

\section{Souches bactériennes de référence}

Pour le contrôle de l'efficacité de l'extraction de l'ADN à partir des selles ainsi que la réaction d'amplification par PCR, sept souches de référence ont été utilisées (C. jejuni ATCC 29428, C. coli ATCC 33559, C. coli CIP 7080, C. jejuni CIP 702, C. fetus CIP 5396, C. upsaliensis CIP 103681 et C. hyointestinalis CIP 104686).

\section{Réaction de polymérisation en chaîne (PCR)}

La recherche de Campylobacter dans les selles a été réalisée par PCR en utilisant en premier lieu des amorces spécifiques à Campylobacter toutes espèces. Ensuite l'identification des isolats au rang des espèces (C. jejuni, C. coli, C. fetus, C. upsaliensis et C. hyointestinalis) a été effectuée en utilisant des amorces spécifiques. La liste des amorces, les gènes cibles, la température d'hybridation ainsi que la taille des amplicons sont présentés dans le tableau 1.

Les réactions de PCR ont été effectuées dans un volume total de $50 \mu \mathrm{L}$ comprenant $10 \mu \mathrm{Ld}$ 'ADN extrait, $0,2 \mu \mathrm{M}$ de chaque amorce, $200 \mu \mathrm{M}$ de chacun des quatre désoxynucléotides phosphate, 2,5 $\mathrm{mM}$ de $\mathrm{MgCl}_{2}$, $1 \mathrm{X}$ de tampon d'amplification $(50 \mathrm{mM}$ de $\mathrm{KCl}, 10 \mathrm{mM}$ de Tris- $\mathrm{HCl}$ à $\mathrm{pH} 8,3$ ) et 1,5 unité de l'enzyme Taq polymerase recombinant (Invitrogen Life Technologies, Carlsbad, CA, USA). L'amplification a été réalisée dans un thermocycleur (BIO-RAD C1000) en 40 cycles ( $30 \mathrm{~s}$ de dénaturation à $94^{\circ} \mathrm{C}, 40 \mathrm{~s}$ d'hybridation avec une température variable selon les amorces, $40 \mathrm{~s}$ d'élongation à $72^{\circ} \mathrm{C}$ ) après $5 \mathrm{~min}$ de dénaturation à $94^{\circ} \mathrm{C}$. Les produits d'amplification ont été détectés par électrophorèse sur un gel d'agarose à 


\begin{tabular}{|c|c|c|c|c|c|}
\hline Nom des amorces & Séquences des amorces ( $5^{\prime}$ à $\left.3^{\prime}\right)$ & $\begin{array}{l}\text { Espèce détectée } \\
\text { et gène cible }\end{array}$ & $\mathrm{T}_{\mathrm{m}}\left({ }^{\circ} \mathrm{C}\right)$ & $\begin{array}{c}\text { Produit } \\
\text { d'amplification }\end{array}$ & Référence \\
\hline $\begin{array}{l}\text { C412F } \\
\text { C1228R }\end{array}$ & $\begin{array}{l}\text { GGATGACACTITTCGGAGC } \\
\text { CAT TGTAGCACGTGTGTC }\end{array}$ & $\begin{array}{l}\text { Toutes espèces } \\
\text { 16SrRNA }\end{array}$ & 58 & $816 \mathrm{bp}$ & [6] \\
\hline $\begin{array}{l}\text { CJmapAN3F } \\
\text { CJmapAN3R }\end{array}$ & $\begin{array}{l}\text { TGGTGGTTITGAAGCAAAGA } \\
\text { GCTTGGTGCGGATTGTAAA }\end{array}$ & $\begin{array}{l}\text { C. jejuni } \\
\text { mepA }\end{array}$ & 58 & 413 bp & [7] \\
\hline $\begin{array}{l}\text { HYO1F } \\
\text { HYOFET23SR2 }\end{array}$ & $\begin{array}{l}\text { ATAATCTAGGTGAGAATCCTAG } \\
\text { GGGAGTAAATCTTAATACAAAGTTAGG }\end{array}$ & $\begin{array}{l}\text { C. hyointestinalis } \\
23 \mathrm{~S} \text { rRNA }\end{array}$ & 54 & $468 \mathrm{bp}$ & [7] \\
\hline $\begin{array}{l}\text { CCceuEN3F } \\
\text { CCceuEN3R }\end{array}$ & $\begin{array}{c}\text { AAGCGTTGCAAAACTTTATGG } \\
\text { CCTTGTGCGCGTTCTTTATT }\end{array}$ & $\begin{array}{l}\text { C. coli } \\
\text { ceuE }\end{array}$ & 58 & $330 \mathrm{bp}$ & [7] \\
\hline $\begin{array}{l}\text { FETNF } \\
\text { HYOFET23SR2 }\end{array}$ & $\begin{array}{l}\text { CGATAATTGATGTGAGAATCATC } \\
\text { GGGAGTAAATCTTAATACAAAGTTAGG }\end{array}$ & $\begin{array}{l}\text { C. fetus } \\
\text { 23S rRNA }\end{array}$ & 56 & $473 \mathrm{bp}$ & [7] \\
\hline $\begin{array}{l}\text { CHCU146F } \\
\text { CU1024R }\end{array}$ & $\begin{array}{l}\text { GGGACAACACTTAGAAATGAG } \\
\text { CАСТTCCGTATCTCTACAGA }\end{array}$ & $\begin{array}{l}\text { C. upsaliensis } \\
16 \mathrm{~S} \text { rRNA }\end{array}$ & 60 & $878 \mathrm{bp}$ & [6] \\
\hline
\end{tabular}

Tm : melting temperature ou température de demi-dénaturation ; bp : base de paires.

2,5\% avec bromure d'éthidium. Pour chaque réaction de PCR, un contrôle positif a été effectué en utilisant les souches de référence. Pour vérifier la spécificité des amorces utilisées pour l'amplification de Campylobacter, une réaction de PCR contenant l'ADN d'Escherichia coli a été réalisée. Finalement, un contrôle négatif contenant de l'eau au lieu de l'ADN a été utilisé.

\section{Résultats}

Sur les 90 échantillons de selles testés, l'ADN de Campylobacter spp. a été détecté dans 10 échantillons (11,1\%). Les espèces détectées par des amorces spécifiques au rang de l'espèce étaient C. coli et C. jejuni. Deux échantillons fécaux ont été positifs pour $C$. jejuni et
C. coli à la fois. Six échantillons (60\%) qui ont donné un résultat positif en utilisant les amorces spécifiques au genre Campylobacter n'ont pas pu être identifiés au rang de l'espèce avec les amorces spécifiques à C. coli, C. jejuni, C. fetus, $C$. upsaliensis et $C$. hyointestinalis. En revanche, l'ADN de C. fetus, C. upsaliensis et $C$. hyointestinalis n'a été détecté dans aucun des échantillons de selles testés (Tableau 2). Aucun amplicon n'a été obtenu avec le tube PCR contenant l'ADN d'E. coli.

\section{Discussion}

Dans la présente étude, on a cherché par PCR la prévalence des bactéries du genre Campylobacter à partir de 90 échantillons deselles chezdesenfants diarrhéiques au nord du Liban. La

\begin{tabular}{|c|c|}
\hline $\begin{array}{l}\text { Tableau } 2 \text { Répartition d } \\
\text { de selles analysées }\end{array}$ & les échantillons \\
\hline Nombre des échantillo & \\
\hline Espèces identifiées & 4 \\
\hline C. coli & 1 \\
\hline C. jejuni & 1 \\
\hline C. coli + C. jejuni & 2 \\
\hline C. fetus & - \\
\hline C. upsaliensis & - \\
\hline C. hyointestinalis & - \\
\hline Espèces non identifiées & 6 \\
\hline
\end{tabular}

prévalence de Campylobacter spp. était de 11,1\%. Une étude antérieure réalisée au Liban en 1998 sur la prévalence de Campylobacter chez des patients diarrhéiques a montré une prévalence de $0,7 \%(2 / 281)$ [7]. Notre étude a montré une prévalence nettement plus importante. Des études réalisées dans des pays en développement ont montré des prévalences similaires à la prévalence obtenue dans notre étude. La prévalence de Campylobacter dans les diarrhées humaines était de 17,7\% en Algérie [8], 9,9\% au Brésil [9], 9,0 \% en Égypte [10], 8,7\% en Iran [11] et 5,5\% en Jordanie [12]. Une autre étude réalisée à Londres en 1999 a montré que la prévalence de Campylobacter était de $13,16 \%$ [13].

Le taux d'infection à Campylobacter dans le monde entier est en augmentation, avec un nombre de cas dépassant souvent celui des salmonelloses et des shigelloses. Cette augmentation nécessite une meilleure compréhension de l'épidémiologie de la campylobactériose $[14,15]$.

La PCR est considérée comme une technique sensible pour la détection de Campylobacter dans les selles humaines. L'application de la PCR fournit une description rapide et précise de la prévalence de Campylobacter associée aux diarrhées 
humaines [16]. Les inhibiteurs de la PCR d'origine fécale, comme les sels biliaires, des produits de dégradation de l'hémoglobine et des polysaccharides complexes, peuvent être enlevés en utilisant des kits commerciaux comme cela a été fait dans la présente étude. D'autre part, un inconvénient de la méthode de détection par PCR est le manque d'isolats, et donc l'incapacité d'accomplir des tests de sensibilité aux antibiotiques [16].

Parmi les dix échantillons positifs, on a pu identifier les espèces de Campylobacter dans quatre échantillons, alors que six échantillons n'ont pas pu être identifiés au rang de l'espèce avec les amorces spécifiques à C. fetus, C. upsaliensis et C. hyointestinalis. Il est probable que ces échantillons contenaient des espèces du genre Campylobacter autres que celles testées dans cette étude. En effet, le genre
Campylobacter comprend plus de 16 espèces et six sous-espèces [1]. Des espèces atypiques comme $C$. concisus et $C$. lari peuvent être associées à des diarrhées chez l'homme. La détection de tels Campylobacter est peu commune dans les pays industrialisés, mais plus commune dans les pays en développement [3].

Les amorces utilisées étaient spécifiques de Campylobacter car le tube de PCR contenant l'ADN d'E. coli avec les amorces de Campylobacter spp. n'a pas donné d'amplicons.

\section{Conclusion}

AuLiban,la recherche systématique de Campylobacter en coproculture n'est pas obligatoire. Nos résultats doivent encourager les acteurs de la santé au Liban à introduire la recherche de cette bactérie en routine à côté de la recherche de Salmonella et Shigella chez les diarrhéiques afin d'éviter des résultats de coproculture faux-négatifs.

Finalement, cette étude montre que le nord du Liban est très touché par les campylobactérioses intestinales avecune prévalence de $11,1 \%$. Cette prévalence importante est due essentiellement aux problèmes de pollution de l'eau potable et à la consommation de légumes et fruits irrigués par des eaux polluées ainsi qu'à l'absence de mesures d'hygiène alimentaire. La source primaire des infections à $C$. jejuni ou C. coli chez l'homme est supposée être la manipulation ou la consommation de viandes contaminées, en particulier la viande de volaille. Le contrôle de Campylobacter dans la chaîne alimentaire est maintenant devenu une cible majeure des agences en charge de la sécurité alimentaire dans le monde.

\section{Références}

1. Vandamme P. Taxonomy of the family Campylobacteraceae. In: Nachamkin I, Blaser MJ, eds. Campylobacter, 2nd ed. Washington DC, ASM Press, 2000:3-26.

2. Tauxe RV. Epidemiology of Campylobacter jejuni infections in the United States and other industrialized nations. In: Nachamkin I, Blaser MJ, Tompkins LS, eds. Campylobacter jejuni: current state and future trends. Washington DC, ASM Press, 1992:9-19.

3. Lastovica AJ, Skirrow MB. Clinical significance of Campylobacter and related species other than Campylobacter jejuni and $C$. coli. In: Nachamkin I, Blaser MJ, eds. Campylobacter, 2nd ed. Washington DC, ASM Press, 2000:89-120.

4. Friedman CR et al. Epidemiology of Campylobacterjejuni infections in the United States and other industrialized nations. In: Nachamkin I, Blaser MJ, eds. Campylobacter, 2nd ed. Washington DC, ASM Press, 2000:121-138.

5. Inglis GD, Kalischuk LD. Use of PCR for direct detection of Campylobacter species in bovine feces. Applied and Environmental Microbiology, 2000, 69:3435-3447.

6. Linton D, Owen RJ, StanleyJ. Rapid identification by PCR of the genus Campylobacter and of five species enteropathogenic for man and animals. Research in Microbiology, 1996, 147:707-718.

7. Talhouk RS et al. Prevalence, antimicrobial susceptibility and molecular characterization of Campylobacter isolates recovered from humans and poultry in Lebanon. Journal Medical Libanais, 1998, 46:310-316.

8. Megraud F et al. Incidence of Campylobacter infection in infants in western Algeria and the possible protective role of breast feeding. Epidemiology and Infection, 1990, 105:73-78.
9. Mangia AH et al. Aetiology of acute diarrhoea in hospitalized children in Rio de Janeiro City, Brazil. Journal of Tropical Pediatrics, 1993, 39:365-367.

10. Rao MR et al. Pathogenicity and convalescent excretion of Campylobacter in rural Egyptian children. American Journal of Epidemiology, 2001, 154:166-173.

11. Hamidian $M$ et al. fla-typing, RAPD analysis, isolation rate and antimicrobial resistance profile of Campylobacter jejuni and Campylobacter coli of human origin collected from hospitals in Tehran, Iran. Annals of Microbiology, 2011, 61:315-321.

12. Na'was TE, Abo-Shehada MN. A study of the bacterial and parasitic causes of acute diarrhoea in northern Jordan. Journal of Diarrhoeal Diseases Research, 1991, 9:305-309.

13. Lawson AJ et al. Large-scale survey of Campylobacter species in human gastroenteritis by PCR and PCR-enzyme-linked immunosorbent assay. Journal of Clinical Microbiology, 1999, 37:3860-3864.

14. Altekruse SF et al. Campylobacter jejuni, an emerging foodborne pathogen. Emerging Infectious Diseases, 1999, 5:2835.

15. Coker AO et al. Human campylobacteriosis in developing countries. Emerging Infectious Diseases, 2002, 8: 237-244.

16. Maher $\mathrm{M}$ et al. Evaluation of culture methods and a DNA probe-based PCR assay for detection of Campylobacter species in clinical specimens of feces. Journal of Clinical Microbiology, 2003, 41:2980-2986. 